\title{
MARRUECOS Y SU NUEVA POLÍTICA EXTERIOR HACIA ÁFRICA. CONTINUIDADES, DISCONTINUIDADES Y PERSPECTIVAS
}

\author{
Morocco and its nem Foreign Policy in Africa. \\ Continuities, Discontinuities and Future Prospects
}

\author{
Blanca Camps-Febrer \\ Doctoranda en Relaciones Internacionales (Universitat Autònoma de Barcelona) \\ E-mail: blanca.camps@uab.cat \\ Óscar Mateos \\ Profesor Asociado e Investigador Principal del grupo de investigación GLOBALCODES \\ de la Facultad de Comunicación y Relaciones Internacionales Blanquerna (Universitat Ramon Llull) \\ E-mail: oscarmm2@blanquerna.url.edu \\ @oscarmateos 1
}

0 Autores

En los últimos años, Marruecos ha efectuado un notable giro en su agenda exterior hacia África. De la mayor discreción que ha caracterizado la posición del régimen alauí en las últimas tres décadas, el país ha pasado a poner en marcha una serie de decisiones que indican que algo muy de fondo podría estar cambiando en la política internacional marroquí hacia esta región. Sin duda, el retorno a la Unión Africana (UA), en febrero de 2017, o la demanda formal de adhesión a la CEDEAO/ECOWAS (organización que agrupa a los Estados de la región de África Occidental), pocos meses después, son decisiones de un gran alcance, que indican que África podría erigirse en una de las piedras angulares de la nueva diplomacia marroquí.

El presente artículo tiene tres objetivos principales. En primer lugar, analizar desde una perspectiva histórica las relaciones del régimen marroquí con el conjunto de países del continente africano, especialmente con la región subsahariana. Segundo, entender las causas de fondo que han motivado esta nueva estrategia diplomática, contextualizando también el conjunto de la agenda internacional del Gobierno de Mohamed VI, e incidiendo de manera especial en las implicaciones que esta estrategia tiene para la cuestión del Sáhara Occidental, o bien en lo que respecta al presente y futuro de la UA. Finalmente, el artículo tratará de abordar algunas de las posibles perspectivas e implicaciones que ello puede tener para los principales retos que afronta la región. 
In recent years, Morocco has made a certain shift in its external agenda towards Africa. From the greater discretion that characterised the position of the Alaouite regime in the previous three decades, the country has undertaken a series of decisions that indicate that something very fundamental could be changing in Moroccan international policy towards this region. Undoubtedly, the return to the African Union (AU) in February 2017, or the formal demand for accession to the CEDEAO/ECOWAS (an organisation that brings together the states of the West African region), a few months later, are two far-reaching decisions that indicate that Africa could become one of the cornerstones of the new Moroccan diplomacy.

This article has three main objectives. Firstly, to analyse from a historical perspective the relations of the Moroccan regime with the African countries, especially with Sub-Saharan region. Second, to understand the root causes that have motivated this new diplomatic strategy. We will contextualize the international agenda of the government of Mohamed VI and focus especially on the implications that this strategy has for the issue of Western Sahara, or with regard to the present and future of the AU. Finally, the article will attempt to address some of the possible implications that this may have for the main challenges facing the Sub-Saharan region.

\section{Introducción}

El retorno del Reino de Marruecos a la Unión Africana (UA) tras 33 años de ausencia en dicha organización ha despertado un importante debate regional e internacional sobre la nueva presencia de la dinastía alauí en el conjunto del continente africano. No solo eso, en los últimos años, Marruecos ha emprendido una política exterior hacia África mucho más activa, que le ha llevado, entre otras cosas, a multiplicar sus viajes a distintos países clave de la región subsahariana, a incrementar su implicación en materia humanitaria o en actividades de construcción de paz en algunos de los contextos que sufren mayor conflictividad; o bien, más recientemente, a solicitar incluso el ingreso en una organización subregional como es la CEDEAO/ECOWAS. ¿Cuáles son las motivaciones del histórico reingreso de Marruecos en la UA? ¿Qué factores explican la voluntad del régimen alauí de querer desempeñar un papel más activo en la región? ¿Qué implicaciones tiene este giro tanto para Marruecos como para la región subsahariana y para el conjunto del continente? El objetivo del presente artículo es arrojar luz sobre alguno de estos aspectos, reconociendo la enorme trascendencia y las posibles implicaciones de esta presencia más activa de la política exterior marroquí en el conjunto del continente africano.

Una primera cuestión que el artículo pretende discutir es, precisamente, que la intensa agenda marroquí actual en África no hay que entenderla tanto como resultado de un giro repentino sino, como parte de una estrategia, más o menos continuada, desde la etapa de Hassan II, que ha sido intensificada con la llegada de Mohamed VI, y que presenta determinadas continuidades respecto a etapas anteriores. De este modo, la tradicional "diplomacia económica y religiosa selectiva”, utilizada por Marruecos en las últimas décadas, ha logrado consolidar algunas de las alianzas habituales, a la vez que ha incorporado a otros países del continente hasta hace poco alejados de la órbita del régimen alauí, tales como Nigeria o Etiopía.

Un segundo aspecto que el artículo pretende abordar es que los motivos que han llevado a la profundización de esta agenda marroquí con África son múltiples y cabe entenderlos desde di- 
ferentes niveles. A nivel doméstico, los intereses de las élites económicas marroquíes han sido fundamentales, sobre todo en un contexto de notable crecimiento económico del continente africano que ha sido proyectado, tanto por organismos internacionales como por otros actores de índole internacional (medios de comunicación, think tanks, consultoras internacionales, etc.), como una región en despegue y con gran potencial económico. A nivel geoestratégico, tanto el retorno a la UA como la intensificación de la presencia marroquí en territorio africano tienen que ver con la voluntad de desplegar una estrategia que confía en un papel más activo de Marruecos en las relaciones internacionales africanas y globales; y en particular, que aspira a redibujar su estrategia respecto al Sáhara Occidental. Rabat estaría apostando así por confrontar el papel de la República Árabe Saharaui Democrática (RASD) desde dentro de la UA, tejiendo nuevas alianzas y apoyos respecto a esta cuestión.

Finalmente, cabe entender estos dos aspectos en un contexto regional de cierto cambio en la correlación de fuerzas. La desaparición de Gadafi, sumada al debilitamiento político de actores como Sudáfrica y muy especialmente de Argelia, países que tradicionalmente habían bloqueado el papel marroquí en el continente africano, han sido claves en el reingreso de Marruecos en la UA y explican también su mayor capacidad de movimiento en el conjunto del continente.

El artículo se estructura en tres partes principales. En primer lugar, desde una perspectiva histórica que recorre los regímenes liderados por Mohamed V y Hassan II, se analizan las principales características de la política exterior marroquí en estas etapas hacia el conjunto de países del África. Segundo, el texto trata de entender las causas de fondo que han motivado la intensificación de la estrategia diplomática marroquí hacia el continente, contextualizando también el conjunto de la agenda internacional del Gobierno de Mohamed VI desde un inicio e incidiendo de manera especial en las implicaciones que esta estrategia tiene para la cuestión del Sáhara Occidental, así como para el presente y futuro de la UA. Finalmente, el artículo esboza algunas conclusiones y perspectivas, tanto en clave doméstica como regional.

\section{Breve aproximación a las dinámicas y características de la política exterior marroquí en África}

\section{1. ¿Una "prerrogativa real"?}

El ámbito de la política exterior y los procesos en los que las decisiones políticas se adoptan son habitualmente un terreno sensible y sujeto a múltiples variables y, en ocasiones, a fuentes inaccesibles para el ámbito de la investigación en las relaciones internacionales. En este sentido, la tarea de dilucidar los procesos y actores que intervienen en la configuración de la política exterior marroquí se convierte en un ejercicio, si cabe, más titánico. Según Parejo y Feliu (2013), el Reino de Marruecos es percibido como un régimen neopatrimonial autoritario en el que existe una competencia política limitada y en el que la acumulación de poder está esencialmente concentrada en manos del monarca.

Aunque la transparencia y la rendición de cuentas son aspectos casi inexistentes y la estructura efectiva que influye en el proceso de diseño de políticas no está oficialmente disponible, la mayoría de autores considera que la estructura de la política exterior marroquí es enormemente jerárquica. Por ejemplo, con relación al régimen de Hassan II (1961-1999), El Houdaïgui (2003) considera que existía una "unidad decisional central" compuesta por el rey, el gabinete 
real y algunos miembros pertenecientes a la élite de las Fuerzas Armadas. Durante esta etapa, la decisión última era siempre tomada por el rey y tan solo un grupo de hombres de confianza del monarca tenía acceso y podía influir en sus decisiones. El liderazgo del rey y su personalidad se convertían de este modo en un elemento clave que determinaba las decisiones y la actitud del Estado. Las políticas eran negociadas y adoptadas en el círculo más estrecho de colaboradores, donde la confianza era uno de los aspectos clave. Los asuntos externos del reino se convirtieron oficialmente con la Constitución de 1962 en una "prerrogativa real", donde las decisiones estratégicas eran adoptadas por el rey y sus principales colaboradores (Fernández Molina, 2007; El Katiri, 2015). Como veremos más adelante, en la etapa de Mohamed VI, el gabinete real ha continuado siendo un espacio fundamental, incluso tras la aprobación de la Constitución de 2011, si bien con la incorporación de nuevas figuras (Abouzzohour y Tomé Alonso, 2018).

Este "Gobierno en la sombra" ha sido, a todas luces, más influyente y activo implementador de la política exterior marroquí que el propio ministro de Exteriores. Dicha estructura se solapa con muchas de las áreas oficiales de Gobierno, conviviendo un primer círculo de actores en torno al monarca que son raramente cuestionados, y un segundo círculo de élites que compiten entre ellas y que no tienen capacidad de cuestionar las decisiones del primer círculo (Feliu y Parejo, 2009). En opinión de Fahd Iraqi (2016), existe una notable diferencia en la composición del gabinete real en función de los perfiles y la experiencia de cada uno. Mientras que en la etapa de Hassan II los miembros de este "Gobierno en la sombra" eran esencialmente figuras políticas con un dilatado "pedigree makhzen" ${ }^{1}$, los colaboradores de Mohamed VI son especialistas y expertos en diferentes áreas estratégicas. Dos o tres de estos consejeros reales acompañan habitualmente al monarca en sus visitas al exterior junto con los ministros pertinentes. Como veremos, el gabinete real se ha hecho más visible en los últimos años y sus integrantes son más jóvenes y con un perfil más basado en su expertise (Iraqi, 2016; Abouzzohour y Tomé Alonso, 2018).

Sea como fuere, y teniendo en cuenta lo centralizados que son los procesos de toma de decisiones, otros muchos actores intervienen en el diseño de la política exterior marroquí (El Katiri, 2015). Partidos políticos, asesores militares y de seguridad, empresas y conglomerados privados o semiprivados, agencias estatales, así como líderes religiosos han desempeñado y continúan jugando un papel fundamental en el desarrollo de las políticas marroquíes bajo el paraguas del rey.

\subsection{El "Gran Marruecos" y la política exterior hacia África: no solo un mito fundacional}

Es importante aproximarse a la identidad marroquí como un factor esencial en la conformación de la política exterior nacional. Es parte de la responsabilidad del monarca preservar y defender la unidad del Reino. De hecho, "es el Garante de la independencia del país y de la integridad territorial del Reino dentro de sus fronteras auténticas" (art. 42). Lo que significan estas "fronteras auténticas” está en el centro de la identidad marroquí y del Reino, especialmente en relación con el continente africano.

Uno de los lugares comunes para empezar a entender la historia entre Marruecos y África es la imagen que a Hassan II le gustaba dar de Marruecos, en la que comparaba al país con un

l La palabra makhzen hace referencia al entorno real, a las familias notables y a la élite política y territorial cercana a la casa real y aliada tradicionalmente con esta. 
árbol, cuyas raíces se encuentran en África y sus ramas y hojas se extienden por Europa. Estas raíces africanas se remontaban a los imperios Almorávide y Almohade, imperios fundacionales que sostienen no solo una versión africana del pasado marroquí sino también una visión expansionista justificada de la nación y su papel en la región. Estos lazos regionales y continentales legitiman de forma histórica las actuales estrategias con los tres principales vínculos transaharianos: la religión, el comercio y las migraciones (El Khayat, 2016).

De hecho, más que un mito fundacional, la idea del "Gran Marruecos" intersecciona con una de las dos, superpuestas y hasta cierto punto irreconciliables, concepciones de la identidad marroquí: una basada en el modelo westfaliano de fronteras territoriales y la concepción weberiana de la soberanía estatal, y la otra basada en la Umma islámica y el califa bay’ah. Estas dos concepciones superpuestas de la soberanía desempeñan, como tales, un papel diferente y a la vez complementario: la esencia islámica y tradicional de la monarquía (antiguamente sultanato), y la forma moderna y materialización de la otra esencia fluida y no estática. La ocupación del Marruecos occidental, piedra angular en la configuración del papel de Marruecos en África en las últimas décadas, solo puede ser explicado desde este marco narrativo.

\section{Antecedentes históricos de la política exterior africana de Marruecos}

\subsection{Mohamed V: de la lucha por la liberación nacional al pragmatismo de la era neoliberal}

En 1955, Sidi Mohamed Ben Youssef, muy pronto Mohamed V, regresó a Marruecos de su exilio forzado en Córcega y más tarde en Madagascar. El nuevo monarca había sido exiliado al país malgache por Francia debido a sus visiones nacionalistas. Durante su ostracismo había logrado hacerse con las simpatías de la mayoría del Movimiento Nacional en Marruecos. Cuando se convirtió en rey del Reino de Marruecos, Mohamed V tuvo que coexistir con el Movimiento Nacional, el Istiqlal de Allal Elfassi, un firme partidario del Gran Marruecos. El nuevo contexto puso a Mohamed $V$ en un delicado equilibrio de poder con el movimiento, que todavía mantenía un importante poder material y simbólico en el país (Parejo y Feliu, 2009).

Este movimiento defendía, al menos a nivel retórico, un territorio marroquí que abarcara desde Sudán hasta llegar a Senegal y el río Níger. No obstante, más allá de esta retórica, el entonces primer ministro de Asuntos Exteriores marroquí, el istiqlali Ahmed Balarej, mostró una narrativa mucho más restringida apoyando la lucha de las independencias africanas.

Los primeros años de independencia marroquí se caracterizaron, de este modo, por un fuerte compromiso ideológico y diplomático con las luchas por las independencias alrededor del continente. Los esfuerzos marroquíes por esta cuestión lograron reunir en la Conferencia de Casablanca de enero de 1961 a todos los líderes africanos de izquierda. Junto con Ghana, Guinea, Malí, el Gobierno interino de Argelia, Egipto y Libia, el bloque de Casablanca se convirtió así en un grupo de países que abogaba por la independencia de todas las naciones en el continente y su posterior integración entre ellas. Dicho bloque se desintegró de forma prematura, si bien, junto con el llamado grupo de Monrovia, más moderado, se convirtieron en los impulsores fundacionales de la Organización para la Unidad Africana (OUA) en 1963.

Marruecos participó también en la primera misión de Naciones Unidas en el continente, desplegada en 1960 y 1961 en el Congo (ONUC), así como en otros episodios importantes que te-
Los primeros años de independencia marroquí se caracterizaron por su compromiso con las luchas por las independencias del continente 
nían lugar en territorio africano. Durante esta etapa, Mohamed V concedió asilo y entrenó a los cuadros del Movimiento Popular de Liberación de Angola (MPLA), con la ayuda del entonces líder argelino, Ben Bella; también, acogió a sectores del sudafricano African National Congress (ANC), incluyendo a Nelson Mandela, además de, supuestamente, enviar equipamiento militar a dicho grupo. Este tipo de colaboraciones con movimientos revolucionarios, sin embargo, llegarían pronto a su fin con la llegada de Hassan II y su inequívoco apoyo a los regímenes autoritarios en el continente.

\subsection{Hassan II y la era de la "diplomacia selectiva"}

Tras morir Mohamed V en 1961, la llegada de su hijo y sucesor al trono, Hassan II, no implicó un cambio abrupto de política hacia el continente africano. Ese mismo año, el nuevo monarca ordenaba la creación del Ministerio de Asuntos Exteriores, liderado por el nacionalista y leal a la corona, Abdelkrim El Khatib. De hecho, Hassan II sería uno de los líderes que amenazaría abiertamente con boicotear la Conferencia de El Cairo de la OUA ante la anunciada presencia de Moïse Moshte, líder de Katanga y cómplice del asesinato de Patrice Lumumba. No obstante, Marruecos se convertiría muy pronto en uno de los principales apoyos del Gobierno dictatorial del presidente del entonces Zaire, Mobutu Sese Seko.

La primera Ley Constitucional de 1962, adoptada por referéndum el 7 de diciembre de 1962, reafirmó como objetivo de Marruecos, en tanto que Estado africano, la unidad de África. Como legado de su difunto padre, Hassan II continuaba ensalzando la retórica de la liberación de los pueblos africanos, a la vez que perseguía a la oposición interna y acumulaba paulatinamente poder a nivel doméstico. Su discurso, sin embargo, era cada vez más irreconciliable con la estrategia política y territorial que el régimen estaba desplegando. La llamada Guerra de las Arenas en 1963, que enfrentó a Marruecos y a Argelia, entró abiertamente en conflicto con uno de los principios centrales de la recién creada OUA que reconocía la inviolabilidad de las fronteras poscoloniales (uti possidetis). La reivindicación marroquí sobre las poblaciones de Béchar y Tindouf, integradas en territorio argelino, llevó a numerosos líderes africanos a posicionarse en favor del Gobierno argelino y a deplorar la agresiva actitud del régimen marroquí en esta cuestión.

Tras el contencioso marroquí con Argelia, Marruecos se centró en la excolonia española del Sáhara Occidental. En 1975, el agonizante régimen franquista en España renunció al dominio colonial de dicho territorio mediante los Acuerdos de Madrid². Con el respaldo de EE. UU. y Francia, el Sáhara Occidental fue repartido entre Mauritania y Marruecos, mientras que España lograría retener durante décadas la explotación de un tercio de las minas de fosfatos a través de la empresa FosBoucraa. Mientras que Mauritania abandonaba sus reclamos territoriales sobre este territorio poco después, la ocupación marroquí del Sáhara Occidental llevó al enfrentamiento armado con el Frente Polisario. Tanto la Administración de Carter como la de Reagan en EE. UU. ofrecieron apoyo militar a Marruecos. La ocupación marroquí, así como las denuncias de crímenes de guerra cometidos por las Fuerzas Armadas marroquíes no contribuirían positivamente a la posición del Reino de Marruecos en el conjunto del continente africano. La activa implicación de la OUA en la cuestión del Sáhara Occidental y su liderazgo

2 Los acuerdos de Madrid no son considerados válidos por parte de Naciones Unidas, y a efectos legales, el Sáhara Occidental es el territorio más grande por descolonizar del mundo. Marruecos es, en este marco, la potencia ocupante, sin perjuicio de la responsabilidad de España como antigua potencia colonial. Véase Wrange (2015). 
en promover y monitorear conjuntamente con Naciones Unidas un referéndum libre para la autodeterminación de este territorio ${ }^{3}$ tensarían las relaciones de la organización panafricana con el reino alauí y le llevarían a adoptar un perfil más bajo en el continente.

Sin embargo, la política exterior marroquí hacia África no se evaporó del todo, si bien se resituó, ubicada plenamente en las posiciones del bloque capitalista. El bajo perfil marroquí hacia África se concretó en una política de relaciones y acuerdos bilaterales en cuestiones diversas y conducida a través de los vínculos personales de Hassan II con magnates y líderes autoritarios africanos. Para autores como Abdelaziz Barre (1996) o Fernández-Molina (2013), la política exterior marroquí con África a partir de la década de los setenta acabó convirtiéndose en una política de "bilateralismo selectivo". Marruecos cerraría, a partir de entonces, unos 88 acuerdos bilaterales con diferentes países africanos, en su mayoría francófonos, tales como Gabón, el entonces Zaire o la República Centroafricana, pero también con otros contextos como Guinea Ecuatorial (Barre, 1996, p. 19). Estos acuerdos se centraron fundamentalmente en cuestiones culturales, técnicas o económicas.

En el plano de la seguridad, y en pleno escenario de Guerra Fría donde el continente africano ocupaba un lugar significativo, el régimen de Hassan II desempeñó un papel activo en la estrategia occidental, tanto proporcionando tropas para neutralizar revueltas como mediante asistencia militar a regímenes autoritarios (Wenger, 1982; El Katiri, 2015). Marruecos se erigió en uno de los aliados clave del bloque occidental, tanto para Francia como convirtiéndose en base y punto geoestratégico para EE. UU. en África. En 1977, por ejemplo, Hassan II enviaría miles de efectivos para detener el movimiento secesionista de Katanga y la implicación de Angola en Zaire, en una de las intervenciones proxy más importantes durante la Administración Carter ${ }^{4}$.

\subsection{El abandono de la OUA y el papel de las Hermandades sufíes}

Marruecos continuó desempeñando un papel más o menos activo en la OUA a lo largo de la década de los setenta, si bien el régimen de Hassan II se había enemistado con bastantes de sus miembros. En 1983, el monarca marroquí logró frustrar la Cumbre de Trípoli donde la OUA discutía la admisión de la RASD como miembro de pleno derecho. Un año después, Marruecos y Libia firmaron el Acuerdo de Oujda, ante la sorpresa de muchos por el tradicional apoyo de Gadafi al Frente Polisario y con el consiguiente disgusto de la entonces Administración Reagan. Hassan II demostraba así que en la cuestión del Sáhara Occidental estaba dispuesto a recabar el apoyo de enemigos confesos de Washington. El acuerdo, que duró solo dos años, supuso la retirada del apoyo militar y financiero de Libia al Polisario a cambio de la neutralidad marroquí en la implicación de Trípoli en el Chad.

Pero a pesar de todos los esfuerzos del régimen alauí, existía en el continente un apoyo generalizado a reconocer al Polisario, considerado como un movimiento de liberación de un territorio ocupado. Así fue como, una vez admitido el RASD en la OUA en 1984, el abandono de

3 Ver especialmente el Plan de Paz sobre el Sáhara Occidental adoptado por la OUA en su 19. ${ }^{\text {a }}$ Cumbre en Addis Ababa, 6-12 Junio de 1983 (AHG/Res. 104 [XIX]).

4 Véase a este efecto el informe de inteligencia de la Central Intelligence Agency de los EE. UU. de abril de 1984: Morocco and the United States: Strategic Cooperation after Three Years, Office of Near Eastern and South Asian Analysis. Desclasificado en 2012. En línea: https://www.cia.gov/library/readingroom/docs/CIA-RDP85T00314R000100040002-6.pdf

\section{El bajo perfil} marroquí hacia África se concretó en una política de relaciones y acuerdos bilaterales 
Marruecos se convirtió en algo casi inevitable, teniendo en cuenta que el régimen de Hassan II había construido esta cuestión como un asunto doméstico de vital importancia. La retirada de Marruecos del espacio político regional de mayor relevancia, como era la OUA, supuso una desconexión formal de las dinámicas políticas del continente, pasando a depender de la política de relaciones bilaterales que Hassan II había construido hasta ese momento. Los acuerdos bilaterales selectivos logrados se convertirían a partir de entonces en el canal esencial de la implicación marroquí con África (Barre, 1996), especialmente con las excolonias francófonas y con Guinea Ecuatorial (Fernández-Molina, 2013). Sea como fuere, en el discurso del régimen marroquí, África continuaba siendo una parte integral de sus objetivos, por lo que en Constituciones sucesivas en la etapa de Hassan II, la unidad del continente seguiría siendo el único objetivo explícito que el Estado mencionaba en el preámbulo de la carta magna.

Aunque la cuestión del Sáhara Occidental se había convertido en un obstáculo para el desarrollo de una política marroquí a nivel regional y continental, no supuso el final de la implicación del régimen en el continente. Fue en la década de los ochenta cuando Marruecos decidiría desarrollar nuevos instrumentos de cooperación con África. En un contexto de importante represión interna y de crisis social y económica tras el impacto de los Planes de Ajuste Estructural y de las políticas neoliberales, Hassan II aprobó la creación de una agencia de ayuda al desarrollo (Agence Morocaine de Coopération Internationale) con el objetivo principal de desplegar una política de cooperación sur-sur en África.

Otro de los canales utilizados fue el de las Hermandades espirituales y religiosas sufíes, con fuerte impronta en muchos países de África Occidental, en particular, la Tidjania, cuyo fundador, Ahmed Tidjane, está enterrado en Fez y su tumba es lugar de peregrinaje habitual para muchas personas de países de la región occidental de África. En 1985, por ejemplo, una conferencia de las Hermandades Tidjani celebrada en Fez y que reunió a líderes sufíes de Senegal, Nigeria o del Golfo Pérsico, logró finalizar con un texto de apoyo a las reivindicaciones marroquíes sobre el Sáhara Occidental. La relaciones entre Marruecos y Senegal, en este caso, se fortalecieron a raíz de la apropiación de las organizaciones religiosas Tidjane por parte de los respectivos líderes políticos con el objetivo de promocionar los intereses diplomáticos marroquíes en África Occidental (Sambe, 2010), una práctica de cooptación habitual de Hassan II (Parejo y Feliu, 2013). En 1985 se creó también la Liga de los Ulemas de Marruecos y Senegal con un incremento exponencial de las becas para estudiantes africanos en las universidades marroquíes (Toufiq, 2016).

La decisión de boicotear la OUA derivó así en una estrategia mucho más pragmática que obligó a Rabat a diversificar los instrumentos de política exterior con África. No fue hasta los noventa cuando un posible "retorno" de Marruecos a África se empezó a fraguar. Tal y como señala Fernández-Molina (2013, p. 595), el final de la Guerra Fría contribuyó a la mejora de la imagen de Marruecos en los países no occidentales. El fin de la contienda bipolar propició también una orientación de la política exterior marroquí más pragmática y más centrada en los negocios. A mediados de los noventa, Marruecos tenía más de 150 acuerdos bilaterales con diferentes países africanos. Entre 1996 y 1997 muchos de ellos empezaron a poner fin a su relación con la RASD, entre ellos algunos de los socios marroquíes que más beneficio obtenían de esta relación bilateral con el régimen, tales como Burkina Faso, República del Congo, Santo Tomé y Príncipe, Benín, Guinea-Bissau, Chad, Togo, Suazilandia o Liberia (Fernández-Molina, 2013). La guerra civil en Argelia también supondría una oportunidad de incrementar el poder de Marruecos en la región debido al debilitamiento del régimen argelino 
y su incapacidad de desempeñar un papel tan activo como hasta entonces en el continente (Fernández-Molina, 2013).

\section{Mohamed VI y la nueva estrategia hacia África \\ 4.1. Cambios y continuidades en la política exterior de mohamed VI}

Existe cierta unanimidad en considerar que desde la llegada de Mohamed VI al trono en 1999, las relaciones con África se han convertido en una prioridad de la política exterior marroquí (Hernando de Larramendi y Tomé-Alonso, 2017; Chamkhi y Boutkhili, 2017; El Khayat, 2016). Ya desde un inicio, dicho interés se concretó en acciones y gestos determinados. En el año 2000, el monarca anunciaría en la cumbre África-Unión Europea, su intención de cancelar la deuda externa de los países africanos menos desarrollados (Hernando de Larramendi y ToméAlonso, 2017), o bien llevaría a Rabat a emprender un intenso periplo por diversos contextos con el objetivo de firmar acuerdos bilaterales, siguiendo la estela de la tradicional estrategia diplomática de sus antecesores. De este modo, entre 1999 y 2014, los flujos comerciales entre Marruecos y el conjunto de la región supusieron un incremento anual medio del 14,7 \%, mientras que la inversión directa marroquí en África ha acabado representando un $63 \%$ del total de la inversión del régimen alauí entre 2008 y 2013 (Namane y Gharbaoui, 2017).

Para Tchetchoua Tchokonte (2017), la etapa de Mohamed VI ha supuesto una constante "ofensiva global y multiforme” hacia África que, además, ha implicado un reajuste de sus dispositivos diplomáticos. Dicho autor considera que existen al menos tres dimensiones en esta ofensiva marroquí que merece la pena señalar:

» Una primera dimensión es la de carácter bilateral, que ha priorizado por lo que denomina como una "diplomacia de proximidad". Consciente de su aislamiento en la escena africana (especialmente desde 1984), Marruecos ha priorizado por los vínculos personales con sus socios africanos, apostando por intensificar las visitas bilaterales de carácter oficial y, como hemos señalado, por la firma de contratos y de todo tipo de acuerdos económicos y comerciales.

» Una segunda dimensión de esta ofensiva es la que adopta un carácter multilateral. Su reingreso en la UA o la demanda de admisión en la CEDEAO/ECOWAS vienen precedidas por su papel en la CEN-SAD (Comunidad de Estados Sahelo-Saharianos) que agrupa a un total de 28 países africanos, o por la organización, entre otros eventos y cumbres, de la primera conferencia euroafricana sobre migración y desarrollo que tuvo lugar en Rabat en julio de 2006 o que a finales de 2016 le llevó a albergar la Conferencia de Cambio Climático en Marrakech.

» Finalmente, una tercera dimensión es la de carácter militar y cultural. A nivel militar, Marruecos lleva un tiempo ofreciendo su experiencia a diversos países africanos, especialmente en materia de formación de oficiales dentro de sus propias academias reales. Bajo la bandera de Naciones Unidas, Rabat se ha implicado también de forma activa y regular en la intervención militar en diversos conflictos armados africanos, como por ejemplo en la Misión de Naciones Unidas en la República Democrática del Congo (MONUC), en la operación de Naciones Unidas en Costa de Marfil (ONUCI) o en la misión desplegada en Malí (MINUSMA). Asimismo, el régimen alauí está colaborando con diversos países en las 
estrategias antiterroristas de la región, especialmente contra Boko Haram, convirtiéndose, por su experiencia en este ámbito, en una ayuda clave para Gobiernos como el de Níger. A nivel cultural, a la utilización de los medios de diplomacia religiosa empleados en etapas anteriores, cabría sumar la creciente capacidad de Marruecos de atraer estudiantes africanos mediante la asignación de más de 6.000 becas de estudio cada año.

Los motivos que explican la intensificación de esta política hacia el continente africano son también diversas. En el plano económico, numerosas voces sugieren que existe un cambio de contexto nacional y continental que es fundamental para entender la profundización de esta estrategia, especialmente en los últimos años. África ha ido adquiriendo la condición de espacio potencialmente atractivo y relevante por su aparente despegue económico (la región ha experimentado un crecimiento económico sostenido cercano al $5 \%$ en la última década), y en el que la concurrencia de países emergentes, especialmente de China, pero también de numerosas empresas internacionales, ha situado al continente en la escena económica global, para muchos, de forma inesperada. La nueva retórica del Africa rising ha sido abanderado no solo por Estados y organismos internacionales sino también por medios de comunicación globales que, como en los casos de The Economist o Times, han acabado por conferir al continente la etiqueta de territorio repleto de oportunidades (Mateos, 2015). Ante esta realidad, Marruecos ha optado por aprovechar el potencial político y económico de la región subsahariana (Fabricius, 2017). Para las empresas marroquíes (muchas de ellas de propiedad estatal, parcial o totalmente), la región se ha convertido en un interesante mercado, tras haberse centrado durante mucho tiempo en el mercado europeo, dirigiendo ahora el $40 \%$ de sus inversiones hacia territorio africano (Louw-Vaudran, 2018).

Para Sami El Khayat (2016) existe un elemento clave en toda estrategia y es comprender también que Marruecos está limitado en su integración en el Magreb y en su despliegue hacia Europa, por lo que el conjunto de África se convierte en un espacio clave de expansión internacional para la agenda económica marroquí del presente y del futuro:

En effet l'Afrique est le continent de la croissance et du changement, sa population augmente, la classe moyenne croît rapidement, c'est unes formidable réserve de ressources naturelles et de main d'oeuvre et pour toutes ces raisons elle est destinée à être la dernière frontière de la mondialisation, du développement industriel et de la croissance mondiale. (El Khayat, 2016, p. 70)

A estos cambios en las percepciones sobre la coyuntura económica del continente, cabe sumar una serie de motivaciones históricas, culturales, religiosas e incluso humanitarias que han estado presentes en otras etapas de la política exterior marroquí con África y que ya hemos señalado. A nivel religioso, sin embargo, cabe destacar cómo Mohamed VI ha impulsado, de manera muy especial en su mandato, un liderazgo religioso que aspira a tener una determinada influencia en ciertas regiones del continente. Así, la Fundación para el Ulema Africano, por ejemplo, fue creada en 2015 con el objetivo de "promover, diseminar y consolidar los valores del islam tolerante” (Hernando de Larramendi y Tomé-Alonso, 2017, p. 231). Liderar una visión moderna de la religión y formar imanes de diferentes países africanos como Malí o Burkina Faso, en un contexto donde el radicalismo religioso se ha convertido en una preocupación de primer orden a nivel regional e internacional, es una oportunidad para el régimen alauí de seguir profundizando en una estrategia de influencia y de soft power en el conjunto de la región subsahariana.

Un último elemento que vamos a señalar como relevante en las motivaciones de la estrategia marroquí tiene que ver con una posición más realista del régimen de Mohamed VI respecto a 
esta región. Para algunos autores, el régimen alauí habría constatado que la estrategia de oposición y de "silla vacía" en la UA (antigua OUA) por la presencia de la RASD ha acabado siendo contraproducente, lo que le ha llevado a una visión más pragmática, que permitiría, por un lado, esa mayor presencia e influencia económica en el continente, pero por otro lado también, la posibilidad de ir ganando apoyos desde dentro de la UA para revocar, a medio o largo plazo, la presencia de la RASD en la organización africana (Louw-Vaudran, 2018). Este cambio de posición también se entiende si tenemos en cuenta el papel de la UA en el escenario regional e internacional en tanto que actor ineludible en la prevención de conflictos y en el desarrollo económico, a pesar de todas sus limitaciones y contradicciones. La ausencia marroquí en este foro podría, por lo tanto, suponer un desgaste y un debilitamiento regional e internacional que el régimen alauí preferiría no asumir (Hernando de Larramendi y Tomé-Alonso, 2017). Maruecos quiere ser reconocido como pieza clave en este nuevo escenario de cambio africano y el reingreso en la UA se habría convertido así en un aspecto crucial en dicha estrategia. Cabría preguntarse también si la prioridad en África respecto a lo que el Gobierno marroquí llama la "cuestión nacional" serviría también para legitimar internamente la apuesta por el continente y acallar las voces críticas sobre la apuesta exterior de Marruecos 5 .

\section{2. "It's so good to be back home!": Marruecos y el regreso a la Unión Africana}

El 31 de enero de 2017 el rey Mohamed VI anunció el retorno de Marruecos a la Unión Africana (UA), tras una dilatada ausencia de más de 32 años. Lo hacía en la 28. a cumbre de la UA celebrada en Addis Ababa (Etiopía) y en un discurso que insistía en dos aspectos principales ${ }^{6}$. Por una parte, la idea de que Marruecos no había abandonado nunca África a pesar de la anomalía de dejar su principal organización hacía tres décadas y reivindicando insistentemente la "africanidad" marroquí. Y por otro lado, el monarca desplegaba una lista de acciones, compromisos y tareas que Marruecos ya venía poniendo en marcha y que hacían hincapié en la voluntad de este país de convertirse en un referente político, económico e incluso humanitario en la nueva etapa de la organización. En definitiva, Mohamed VI establecía una relación de interdependencia intrínseca entre su país y el conjunto de la región en un contexto de cambios y que consideraba lleno de oportunidades y posibilidades para ambas partes.

El discurso ante los jefes de Estado africanos, mediante el que Marruecos se incorporaba oficialmente de nuevo a la marcha de la UA, distaba del tono más agrio y duro que unos meses antes el monarca había utilizado en una carta en la que pedía formalmente el reingreso de su país en la organización: "Cuando un cuerpo está enfermo", apuntaba el monarca en la misiva, "es mejor curarlo en el interior que en el exterior del organismo" . Esta idea, como apuntábamos con anterioridad, reflejaba precisamente la estrategia marroquí con respecto a la presencia de la RASD en la UA, apostando a medio y largo plazo por poner en jaque desde dentro la presencia

5 Una de las críticas habituales es hacia la decisión de destinar tantos esfuerzos, diplomacia y recursos hacia África con unos niveles de pobreza y con tantas necesidades por parte de la propia población marroquí. La prueba de que estas críticas han sido tomadas en consideración es el propio discurso del rey del 20 de agosto de 2017 en el cual las nombraba explícitamente.

6 Véase Le discours historique du roi Mohammed VI à Addis Abeba (texte intégral), 31 de enero de 2017, en https://ledesk.ma/2017/01/31/le-discours-historique-du-roi-mohammed-vi-addis-abeba-texte-integral/

7 Véase Message de SM le Roi au 27-ème sommet de l>Union Africaine à Kigali, 17 de julio de 2016, en http:/www.mapnews.ma/fr/activites-royales/message-de-sm-le-roi-au-27-eme-sommet-de-lunion-africaine-kigali 
saharaui en la organización. Más allá de la declaración de intenciones pronunciada en el discurso y de la nueva estrategia en relación con el conflicto del Sáhara Occidental, lo cierto es que toda esta situación podría estar también implicando una cierta reconfiguración de las alianzas políticas dentro del continente.

Para algunos autores, la entrada de Marruecos en la UA - durante mucho tiempo bloqueada por diversos países, especialmente por Sudáfrica y Argelia- se ha debido a una serie de factores que acabaron configurando una coyuntura propicia al reingreso. Además de los efectos de una dilatada e intensa "ofensiva multiforme", siguiendo la idea de Tchetchoua Tchokonte (2017), que tenía en la diplomacia económica y religiosa selectiva uno de sus principales instrumentos de largo alcance y que llevó en los meses previos a un nuevo e intenso tour de Mohamed VI por diversos países africanos (Hernando de Larramendi y Tomé-Alonso, 2017), el cambio de actitud de muchos países, hasta hace poco más beligerantes con la entrada de Marruecos, fue también un elemento clave. Para Louw-Vaudran (2018), el contexto de crecimiento y oportunidades económicas en el continente africano habría llevado, no solo a Marruecos sino a muchos otros países africanos, a adoptar una actitud mucho más pragmática, alejada del espíritu anticolonial que en otros momentos había hecho de su apoyo al Frente Polisario un objetivo irrenunciable. De este modo, países como Nigeria, Etiopía, Tanzania o Ruanda con los que Marruecos ya había celebrado cumbres bilaterales e incluso acordado la puesta en marcha de ambiciosos proyectos de infraestructuras y comerciales ${ }^{8}$, han pasado a adoptar una posición que, sin dejar de apoyar oficialmente a la RASD, no contempla el bloqueo de la nueva presencia marroquí.

El caso de Nigeria es especialmente significativo. Por una parte, su posición más neutral respecto a la entrada de Marruecos estaría influenciada por el resurgir del movimiento secesionista de Biafra, llevando al Gobierno de Abuja y a otros países africanos a una visión menos favorable a movimientos y proyectos secesionistas (Louw-Vaudran, 2018). Chamkhi y Boutkhili (2017) consideran que el Gobierno nigeriano del actual presidente Buhari marcaría, también a partir de 2015, un punto de inflexión en una relación que antes era mucho más estrecha con el Frente Polisario. Esta nueva coyuntura habría significado "una brecha en el eje Alger-Abuja-Pretoria” respecto a la cuestión marroquí que habría sido clave en este nuevo contexto. Un caso similar es el de Senegal, país tradicionalmente más cercano a Rabat. Los fuertes vínculos políticos y, sobre todo, religiosos, llevaron al presidente senegalés, Macky Sall, a desempeñar un papel importante en la negociación final que discutía la entrada del régimen de Rabat, que finalmente fue avalada por los dos tercios necesarios (39 de los 54 Estados africanos) exigidos por la carta de la UA.

Nada de esto se podría tampoco explicar sin el creciente debilitamiento de los países en los que la RASD se había apoyado tradicionalmente, como son Argelia y Sudáfrica, pero también otros como Zimbabue. Mientras que estos dos últimos han sido escenario de sendas crisis políticas, que incluso han llevado a la destitución de los líderes que ostentaban el poder en ambos contextos, en Argelia, la grave situación de salud de su mandatario, Abdelaziz Bouteflika, ha sumido
El regreso a la UA podría implicar una cierta reconfiguración de las alianzas políticas dentro del continente

8 Entre otros acontecimientos y acuerdos de relevancia, el presidente ruandés, Paul Kagame, visitó Marruecos en junio de 2016, con quien firmó un gran número de acuerdos bilaterales. Marruecos también celebró una reunión bilateral con el Gobierno etíope en noviembre de 2016, con presencia de una amplia delegación de negocios marroquí, y en la que se anunció la construcción de una planta fertilizadora que proporcionará a Etiopía lo necesario para producción agrícola. Con el Gobierno nigeriano, Rabat acordó la construcción de un oleoducto masivo que podría beneficiar a diversos países de la subregión. 
al país en una situación de incertidumbre que ha contribuido al fortalecimiento del papel de Marruecos.

Más allá de este cambio en las posiciones y en la correlación de fuerzas que ha tenido lugar en el conjunto del continente, lo cierto es que la UA, con la entrada de Marruecos, afronta una nueva etapa, en la que podrían acontecer diversos escenarios: un primer escenario caracterizado por la tensión y en el que Rabat utilizaría sus instrumentos diplomáticos para impulsar una eventual expulsión del RASD a medio y largo plazo; o bien otro contexto en el que la UA podría favorecer nuevos espacios de negociación y de resolución de este contencioso, ofreciendo la oportunidad a Marruecos de erigirse en un país de referencia dentro de la organización.

\section{Conclusiones}

La política exterior marroquí en África de los últimos años viene marcada por cambios evidentes, pero también por continuidades históricas. Los vínculos económicos y religiosos, que se remontan siglos atrás, junto a una red de relaciones personales que los dos últimos reyes han ido tejiendo, especialmente en África occidental, representan la continuidad principal. En el contexto actual, Marruecos ha seguido utilizando esos vínculos y se ha presentado al mundo como un socio económico de peso, un hub regional estable y de confianza, en un contexto global en el que África es entendida como oportunidad para las potencias tradicionales y emergentes.

Por otra parte, el artículo ha puesto de relieve algunas discontinuidades que han sido claves para explicar la nueva presencia marroquí en África. Con la llegada de Mohamed VI, la política exterior marroquí en África ha ganado en pragmatismo, llevando a Rabat a consolidar las alianzas tradicionales de África occidental y a ampliarlas a nuevos países del conjunto del continente hasta hace poco alejados de la órbita del régimen alauí (como son Nigeria, Ruanda o Tanzania). Esta estrategia ha utilizado los habituales instrumentos de diplomacia económica selectiva en un ambiente de crecimiento económico africano, y en un contexto, y este hecho va a ser determinante, de aparente debilitamiento de los principales adversarios de Marruecos en el continente, como son Sudáfrica y, muy especialmente, Argelia.

La nueva implicación en la escena multilateral africana supone también para Marruecos un cambio de estrategia respecto al Sáhara Occidental, factor que le llevó a abandonar la entonces OUA en 1984. Fruto de esa actitud más pragmática, Rabat habría preferido hacer frente al RASD desde dentro de la organización, entendiendo que la política de "silla vacía" seguida hasta ahora le supone muchos más problemas que oportunidades y quizás considerando que, a medio y largo plazo, podría ser capaz de seducir a algunos actores africanos para lograr la expulsión del RASD de la UA. La nueva presencia en la UA también podría erigir a Rabat en un actor con creciente peso e influencia en las decisiones y en el devenir de la organización regional, en un contexto marcado por una agenda internacional preocupada por la seguridad en la región y por la lucha antiterrorista. 


\section{Referencias}

Aït Akdim, Y. (2016, 29 de abril). La Tidjaniyya, arme secrete du soft power marocain en afrique. Le Monde. Recuperado de http:/www.lemonde.fr/international/article/2016/04/29/ la-tidjaniyya-arme-secrete-du-soft-power-marocain-en-afrique_4911069_3210.html

Barre, A. (1996). La politique marocaine de coopération en Afrique. Essai de bilan. En A. Saaf (Ed.), Le Maroc et l'Afrique après l'Indépendance. Série: Colloques et Séminaires, n. ${ }^{\circ} 4$. Université Mohammed V. Rabat: Institut des Etudes Africaines.

Barre, A. (2004). Les rélations entre le Maroc et les pays de l'Afrique Sub-saharienne. En L. Marfaing \& Steffen Wippel (Dirs.), Les relations transsahariennes à l'époque contemporaine (pp. 61-88). París y Berlin: Éditions Karthala et ZMO.

Bouhout, M. R. (1996). La politique marocaine de coopération avec l'Afrique: subsaharienne 1960-1994. En A. Saaf (Ed.), Le Maroc et l'Afrique après l>Indépendance. Série: Colloques et Séminaires, n. ${ }^{\circ}$ 4. Université Mohammed V. Rabat: Institut des Etudes Africaines.

ElHoudaïgui, R. (2003). La politique étrangère sous le règne de Hassan II. Paris: L'Harmattan.

ElKhayat, S. (2016). Les enjeux de la politique africaine du Maroc. Revue Espaces Géographiques et Société Marocaine (15).

Edzimbi, F. X. N. (2017). La maturité stratégique comme élément d'affirmation d'un état sur la scène internationale : l'exemple du Royaume du Maroc. Revue Espaces Géographiques et Société Marocaine (19).

Feliu, L., \& Parejo, M. ${ }^{a}$ A. (2009). Marruecos: la reinvención de un sistema autoritario. En F. Izquierdo Brichs, Poder y Regímenes en el Mundo Árabe Contemporáneo. Interrogar la actualidad. Serie Mediterráneo y Oriente Medio. Fundació CIDOB. Barcelona: Edicions Bellaterra, S.L.

Fernández-Molina, I. (2007). Le PJD et la Politique Étrangère du Maroc: Entre l'idéologie et le pragmatisme. Mediterráneo, (7). Barcelona: CIDOB edicions.

Fernández Molina, I. (2013). La política exterior de Marruecos en el reinado Mohamed VI, (19992008): actores, discursos y proyecciones internas. Tesis. Recuperado de http://eprints.ucm. es/21808/1/T34552.pdf

Iraqi, F. (2016, 21 de junio). Maroc: qui sont les conseillers du cabinet royal de Mohammed VI? Jeune Afrique. Recuperado de http://www.jeuneafrique.com/mag/332768/politique/ maroc-conseillers-cabinet-royal-de-mohammed-vi/

Iraqi, F. (2016, 21 de junio). Omar Kabbaj: le conseiller Afrique de Mohammed VI. Jeune Afrique. Recuperado de http://www.jeuneafrique.com/mag/334014/politique/omar-kabbajexpertise-africaine

Kosnett, P. S. (S. F.). Cobras for Hassan. Harvard International Review, 16.

Louw-Vaudran, L. (2018). The meaning of Morocco's return to the African Union. Institute for Security Studies.

Mateos, O. (2015). From Tragedy to Miracle? Africa in a multipolar context. Barcelona: CCJ. 
Parejo Fernández, M. A., \& Feliu, L. (2013). Identidad y regionalización: los actores políticos marroquíes ante la reforma constitucional de 2011. Revista de Investigaciones Políticas y Sociológicas, 12(2), 109-126.

Ojeda-García, R., Fernández-Molina, I., \& Veguilla, V. (2017). Global, Regional and Local Dimensions of Western Sahara's Protracted Decolonization. New York: Palgrave Macmillan. DOI: https://doi.org/10.1057/978-1-349-95035-5

Saaf, A. (Coord.). (1996). Le Maroc Et L>Afrique Après L>Independance. Rabat: Institut Des Etudes Africaines et Le Departement de Droit Publique, Universidad Mohamed V.

Sambe, B. (2010). Tidjaniya: usages diplomatiques d`une confrérie soufie. Politique étrangère, (4), 843-854. DOI: https://doi.org/10.3917/pe.104.0843

Toufiq, A. (2016, 9 de febrero). Les Relations entre le Maroc et les Pays d'Afrique de l'Ouest (discurso). Ministerio del Habous y de Asuntos Islámicos. Recuperado de http://www. habous.gov.ma/fr/documents-de-la-rencontre/3151-les-relations-entre-le-maroc-et-lespays-d\%E2\%80\%99afrique-de-1\%E2\%80\%99ouest,-allocution-de-mr-ahmed-toufiq. html

Tchetchoua Tchokonte, S. (2017). La politique de puissance du Maroc en Afrique subsaharienne. Revue Espaces Géographiques et Société Marocaine, (19).

Wainscott, A. M. (2018). Religious Regulation as Foreign Policy: Morocco's Islamic Diplomacy in West Africa. Politics and Religion, (11), 1-26. DOI: https://doi.org/10.1017/ S1755048317000591

Wenger, M. (1982, mayo). Reagan Stakes Morocco in Sahara Struggle. MERIP Reports, (105). Reagan Targets the Middle East, 22-26, 30.

Wrange, P. (2015, junio). Occupation/annexation of a territory: Respect for international humanitarian law and human rights and consistent EU policy. Directorate-General for External Policies. Policy Department. European Parliament.

Wibisono, A. T. M. (2009). Political elites and foreign policy : democratization in Indonesia. Tesis doctoral. Graduate School of legal studies, Faculty of Law, Leiden University. Recuperado de http://hdl.handle.net/1887/14035 